\title{
Model of Dynamical Correlation in Two-Ionic Strong Coupling Plasmas
}

\author{
T. Chohra, K. Chenini, M.T. Meftah* and A. BoukraA \\ Laboratoire des Energies Nouvelles et Renouvelables dans les Zones Arides et Sahariennes (LENREZA) \\ Faculté des Sciences et Sciences de l'Ingénieur, Université de Ouargla, 30000 Ouargla, Algeria
}

(Received May 7, 2007; in final form March 27, 2009)

\begin{abstract}
The dynamics of an impurity ion of charge $q_{0}$ embedded in a two-component ionic plasma is represented as that of a particle in a random medium. The effect of the surroundings on the impurity is represented by a memory function whose form is proposed in this work. Our choice stands for the strongly coupled plasma for which the memory function has an oscillatory behavior with the plasma frequency. The model therefore describes the plasma in the strong coupling limit. We first derive a master equation governed by this memory function and, with the help of the Laplace transform, we solve it via a quartic algebraic equation. We calculate in the end the dynamical properties, i.e. the autocorrelation functions which are very useful in many areas of plasma physics as in radiative transport and in spectral line shape broadening theories.
\end{abstract}

PACS numbers: 52.20.Dq, 52.65.Cc, 47.40.Nm, 05.20.-y

\section{Introduction}

Equilibrium autocorrelation functions are of great importance in the evaluation of the relaxation time in many problems of physics [1-3]. This time represents the duration of the memory effect on the process under investigation [4-7]. In order to take into account the effect of an observable $A$ on an observable $B$, we must know the ratio between the relaxation times of the equilibrium autocorrelation functions $(A A)$ and $(B B)$. This ratio will then help in telling us what model is a good candidate for representing the process. The calculation of equilibrium autocorrelation functions is based on the master equation [8]. The latter depends on the memory function $M(t)$ [9] which plays an important role in the elaboration of the model. By using an appropriate memory function, we present in this work a model for calculating equilibrium autocorrelation functions relating to the electric microfield and to the velocity of an ion which is valid for the case of a strongly coupled plasma.

\section{The model}

The system considered here is an impurity ion of mass $m_{0}$ and charge $q_{0}$ in equilibrum with a fully ionized plasma of structureless point ions. The equilibrum autocorrelation functions of the electric field and the velocity are defined, respectively, by [9]

\footnotetext{
* corresponding author; e-mail: mewalid@yahoo.com
}

$$
\begin{aligned}
& C(t)=\langle\boldsymbol{E}(t) \cdot \boldsymbol{E}(0)\rangle /\left\langle\boldsymbol{E}(0)^{2}\right\rangle \\
& \equiv\langle\boldsymbol{E}(t) \cdot \boldsymbol{E}\rangle /\left\langle E^{2}\right\rangle, \\
& D(t)=\left\langle\boldsymbol{V}_{0}(t) \cdot \boldsymbol{V}_{0}(0)\right\rangle /\left\langle\boldsymbol{V}_{0}^{2}(0)\right\rangle \\
& \equiv\left\langle\boldsymbol{V}_{0}(t) \cdot \boldsymbol{V}_{0}\right\rangle /\left\langle V_{0}^{2}\right\rangle,
\end{aligned}
$$

where the brackets $\langle\ldots\rangle$ denote an equilibrium Gibbs ensemble average (the canonical one here), and $\boldsymbol{V}_{0}$ is the impurity ion velocity. The correlation function $C(t)$ measures the fluctuation in a collective property of the two-component ionic plasma (TCIP) [8], while $D(t)$ measures the fluctuation in a property of the impurity ion. Besides, these are directly related via the first Newton law by

$$
\frac{\mathrm{d}^{2} D(t)}{\mathrm{d} t^{2}}=-\omega_{0}^{2} C(t)
$$

with

$$
\omega_{0}^{2}=\left(\beta q_{0}^{2} / 3 m_{0}\right)\left\langle E^{2}\right\rangle,
$$

and the function $D(t)$ is given by the master equation

$$
\frac{\mathrm{d}^{2} D(t)}{\mathrm{d} t^{2}}+\omega_{0}^{2} D(t)+\int_{0}^{t} \mathrm{~d} \tau M(t-\tau) \frac{\mathrm{d} D(\tau)}{\mathrm{d} \tau}=0 .
$$

The initial conditions on $D(t)$ are

$$
D(t=0)=1, \quad \dot{D}(t=0)=0
$$

and

$$
M(0)=\omega_{1}^{2}-\omega_{0}^{2}, \quad \omega_{1}^{2}=\left\langle\dot{E}^{2}\right\rangle /\left\langle E^{2}\right\rangle,
$$

where $\beta=1 / k_{\mathrm{B}} T$ and $\dot{E}$ means the time derivative of $E(t)$ at $t=0$. Equation (5) describes the impurity ion dynamics as oscillations in a viscoelastic medium, 
where the characteristic frequency is $\omega_{0}$ and shows a frequency dependent damping given by the Fourier transform of $M(t)$. All many-body effects of the medium on the impurity that are not explicit in (5) are contained in the detailed form of $M(t)$. Our fundamental assumption here is that it is sufficient to include only the magnitude of this function through its exact initial value $M(0)=M_{0}$. Furthermore, this function $M(t)$ must take into account of plasma oscillation and the damping through two time scales $\Omega^{-1}=\omega_{\mathrm{p}}^{-1}$ the inverse of plasma frequency) and $\lambda^{-1}$.

Making use of the results presented in [10] (Eq. (30)), we assume the following form of $M(t)$ :

$$
M(t)=M_{0} \mathrm{e}^{-\lambda t} \cos \Omega t,
$$

where $\Omega \gg \lambda$. For all parameters we used in our calculations, the condition $\Omega \gg \lambda$ is always satisfied. The parameter $\lambda$ is fixed by the Green-Kubo expression for the self-diffusion coefficient $D$ in terms of the velocity autocorrelation function as $[8,10]$ :

$$
\begin{aligned}
& \beta m_{0} D=\int_{0}^{\infty} \mathrm{d} t D(t), \\
& \lambda=\left(\frac{\omega_{1}^{2}}{\omega_{0}^{2}}-1\right) / \beta m_{0} D .
\end{aligned}
$$

The Laplace transform of Eq. (5) gives

$$
\begin{aligned}
& \int_{0}^{\infty} \mathrm{e}^{-z t} \frac{\mathrm{d}^{2} D(t)}{\mathrm{d} t^{2}} \mathrm{~d} t+\omega_{0}^{2} \widehat{D}(z) \\
& +\widehat{M}(z) \int_{0}^{\infty} \mathrm{e}^{-z t} \frac{\mathrm{d} D(t)}{\mathrm{d} t} \mathrm{~d} t=0 .
\end{aligned}
$$

Using the previous initial conditions of $\mathrm{D}(\mathrm{t})$, we find that the solution of (11) can be expressed as

$$
\widehat{D}(z)=\frac{z+\widehat{M}(z)}{z^{2}+z \widehat{M}(z)+\omega_{0}^{2}},
$$

where $\widehat{M}(z)$ is the Laplace transform of the memory function $M(t)$ given as

$$
\begin{aligned}
& \widehat{M}(z)=M_{0} \int_{0}^{\infty} \exp (-z t-\lambda t) \cos (\Omega t) \mathrm{d} t \\
& \quad=\frac{M_{0}(z+\lambda)}{(z+\lambda)^{2}+\Omega^{2}} .
\end{aligned}
$$

At the end, we find that the parameter $\Omega$ satisfies $[8,10]$ :

$$
\begin{aligned}
& \beta m_{0} D=\lim _{z \rightarrow 0} \widehat{D}(z)=\frac{\lambda M_{0}}{\omega_{0}^{2}\left(\Omega^{2}+\lambda^{2}\right)}, \\
& \Omega^{2}=\frac{M_{0}}{\beta m_{0} D \omega_{0}^{2}} \lambda-\lambda^{2}
\end{aligned}
$$

and that the velocity autocorrelation function transform is

$$
\widehat{D}(z)=\frac{z(z+\lambda)^{2}+z \Omega^{2}+(z+\lambda) M_{0}}{z^{4}+2 \lambda z^{3}+z^{2}\left(\Omega^{2}+\lambda^{2}+\omega_{0}^{2}+M_{0}\right)+z\left(M_{0} \lambda+2 \Omega^{2} \lambda\right)+\omega_{0}^{2}\left(\Omega^{2}+\lambda^{2}\right)} .
$$

Equations (5)-(7) and (9) define the approximate model for $D(t)$ and, through Eq. (3), the electric field autocorrelation function. Furthermore, the exact time integrals of $C(t)$ and $D(t)$ are insured through (5) and (8). The input data $\omega_{0}, \omega_{1}$, and $D$ might be taken directly from computer simulation or by an other suitable model.

It is then straightforward to solve (5) by a Laplace transformation, yielding $D(t)$ and $C(t)$ as the sum of four exponentials

$$
\begin{aligned}
& D(t)=\sum_{i=1}^{4} D_{i} \mathrm{e}^{z_{i} t}, \\
& C(t)=\sum_{i=1}^{4} C_{i} \mathrm{e}^{z_{i} t}
\end{aligned}
$$

where the coefficients $D_{i}$ and $C_{i}$ are given by

$$
\begin{aligned}
D_{i} & =-\left(\omega_{0} / z_{i}\right)^{2} C_{i}, \\
C_{1} & =\frac{z_{1}\left(z_{1}+\lambda\right)^{2}+z_{1} \Omega^{2}}{\left(z_{1}-z_{2}\right)\left(z_{1}-z_{3}\right)\left(z_{1}-z_{4}\right)}, \\
C_{2} & =\frac{z_{2}\left(z_{2}+\lambda\right)^{2}+z_{2} \Omega^{2}}{\left(z_{2}-z_{3}\right)\left(z_{2}-z_{4}\right)\left(z_{2}-z_{1}\right)}, \\
C_{3} & =\frac{z_{3}\left(z_{3}+\lambda\right)^{2}+z_{3} \Omega^{2}}{\left(z_{3}-z_{4}\right)\left(z_{3}-z_{2}\right)\left(z_{3}-z_{1}\right)},
\end{aligned}
$$

$$
C_{4}=\frac{z_{4}\left(z_{4}+\lambda\right)^{2}+z_{4} \Omega^{2}}{\left(z_{4}-z_{1}\right)\left(z_{4}-z_{2}\right)\left(z_{4}-z_{3}\right)}
$$

and the $\left\{z_{i}\right\}$ are solutions of the quartic equation (Appendix)

$$
\begin{aligned}
& z^{4}+2 \lambda z^{3}+z^{2}\left(\Omega^{2}+\lambda^{2}+\omega_{0}^{2}+M_{0}\right) \\
& \quad+z\left(M_{0} \lambda+2 \Omega^{2} \lambda\right)+\omega_{0}^{2}\left(\Omega^{2}+\lambda^{2}\right)=0 .
\end{aligned}
$$

Depending on the values of $\lambda, \omega_{0}$, and $\omega_{1}$, the solutions may be either real or complex. In principle, this theoretical analysis applies for arbitrary interaction potentials and plasma composition. We intended that this work is of great interest and should provide a very powerful tool to investigate many dynamic properties and transport phenomena in ionized media such as a plasma and electrolytic solutions. Note that the electric autocorrelation function $C(t)$ in Eq. (18) satisfies the initial conditions $C(0)=1, \dot{C}(0)=0$, the same being true for $D(t)$ as shown in Fig. 1 for the indicated parameters.

\section{Discussion}

In this paper we have presented a theoretical model for dynamical correlation functions in a two-component plasma. The implementation of the plasma oscillations is achieved by a proposed form of the memory function. 


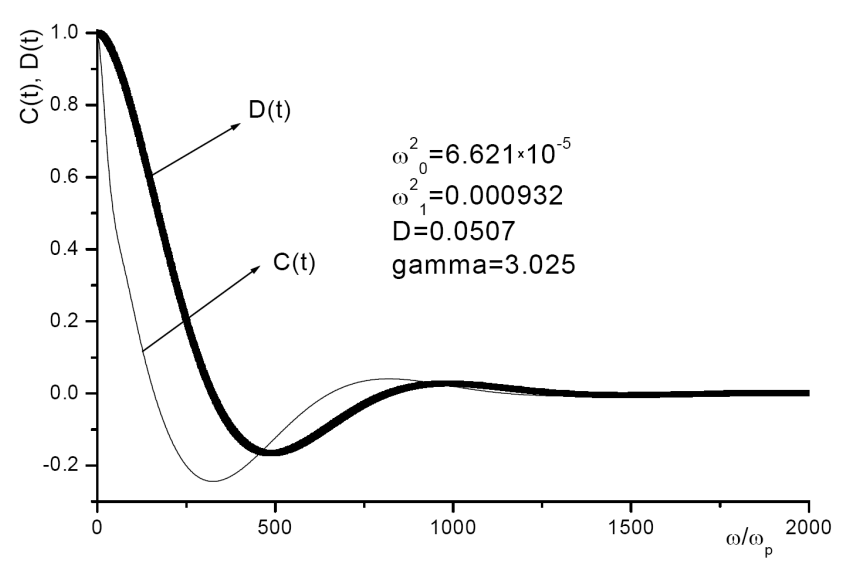

Fig. 1. Relaxation of the correlation function $C(t)$ and $D(t)$ for $100 \% \mathrm{Ar}^{17+}$, where the parameters $\omega_{0}$ and $\omega_{1}$ are given in units of plasma frequency $\omega_{\mathrm{p}}=$ $\left(4 \pi n_{\mathrm{e}} \mathrm{e}^{2} / m_{0}\right)^{1 / 2}$, the time $t$ is in the inverse plasma frenquency units and $n_{\mathrm{e}}$ is the electron density. The coefficient $D$ is given in unit $\omega_{\mathrm{p}} a^{2}\left(a=(3 n / 4 \pi)^{1 / 3}\right)$.

The oscillation modes are calculated by solving a quartic algebraic equation. The solutions are complex (two conjugates of two) except in the limit of high temperatures (then the solutions become real and no oscillations in the correlation function are observed) where the model must be dropped in favor of more adequate models [8].

\section{Appendix: The solution of the fourth-order algebraic equation}

The solution of the fourth-order algebraic equation

$$
z^{4}+a z^{3}+b z^{2}+c z+d=0
$$

is based on the resolution of a cubic equation.

By using the variable change $z=x-\frac{a}{4}$, Eq. (A1) takes the form

$$
x^{4}+p x^{2}+q x+r=0
$$

with

$$
p=b-\frac{3 a^{2}}{8}, \quad q=c-\frac{a b}{2}+\frac{a^{3}}{8}
$$

and

$$
r=d-\frac{a c}{4}+\frac{a^{2} b}{16}-3\left(\frac{a}{4}\right)^{4} .
$$

We look for a positive root $y_{0}$ of the cubic equation

$$
y^{3}+\alpha y^{2}+\beta y+\lambda=0
$$

with

$$
\alpha=\frac{p}{2}, \quad \beta=\frac{1}{4}\left(\frac{p^{2}}{4}-r\right) \text { and } \lambda=-\left(\frac{a}{8}\right)^{2} .
$$

Next, one calculates the following quantities:

$$
\varphi=\sqrt{y_{0}}, \quad \omega_{1}=\sqrt{-y_{0}-\frac{p}{2}+\frac{9}{4 \varphi}} ;
$$

$$
\omega_{2}=\sqrt{-y_{0}-\frac{p}{2}-\frac{9}{4 \varphi}} .
$$

The parameters $\omega_{1}$ and $\omega_{2}$, which can be real or complex, give the roots of Eq. (A2) as

$$
\begin{aligned}
& x_{1}=-\varphi+\omega_{1}-\frac{a}{4}, \\
& x_{2}=-\varphi-\omega_{1}-\frac{a}{4}, \\
& x_{3}=\varphi+\omega_{2}-\frac{a}{4}, \\
& x_{4}=\varphi-\omega_{2}-\frac{a}{4} .
\end{aligned}
$$

For the resolution of the cubic Eq. (A3):

$$
y^{3}+\alpha y^{2}+\beta y+\lambda=0,
$$

one calculates the following quantities:

$$
\begin{aligned}
& p^{\prime}=\frac{\beta}{3}-\left(\frac{\alpha}{3}\right)^{2}, \quad q^{\prime}=2\left(\frac{\alpha}{3}\right)^{3}-\frac{\alpha \beta}{3}+\lambda, \\
& r^{\prime}=q^{\prime 2}+4 p^{\prime 3}, \\
& s=\sqrt[3]{\frac{q^{\prime}+\sqrt{r^{\prime}}}{2}}+\sqrt[3]{\frac{q^{\prime}-\sqrt{r^{\prime}}}{2}}, \\
& t=\sqrt[3]{\frac{q^{\prime}+\sqrt{r^{\prime}}}{2}}-\sqrt[3]{\frac{q^{\prime}-\sqrt{r^{\prime}}}{2}} .
\end{aligned}
$$

1) if $r^{\prime}>0$, the equation admits a real root and two imaginary roots

$$
\begin{aligned}
& y_{1}=s-\frac{\alpha}{3}, \quad y_{2}=\frac{s}{2}+\mathrm{i}\left(\frac{t \sqrt{3}}{2}\right), \\
& y_{3}=\frac{s}{2}-\mathrm{i}\left(\frac{t \sqrt{3}}{2}\right),
\end{aligned}
$$

2) if $r^{\prime}=0$ and $p^{\prime}=0$, the equation admits a triple real root

$$
y=-s-\frac{\alpha}{3},
$$

3) if $r^{\prime}=0$ and $p^{\prime} \neq 0$, the equation admits two real roots

$$
y_{1}=-s-\frac{\alpha}{3}, \quad y_{2}=\frac{s}{2}-\frac{\alpha}{3}, \quad y_{2}=y_{3},
$$

4) if $r^{\prime}<0$, the equation admits the three real roots

$$
\begin{aligned}
& y_{1}=-2 \sqrt{-p^{\prime}} \cos (\theta), \\
& y_{2}=-2 \sqrt{-p^{\prime}} \cos \left(\theta+120^{\circ}\right), \\
& y_{3}=-2 \sqrt{-p^{\prime}} \cos \left(\theta+240^{\circ}\right),
\end{aligned}
$$

where

$$
\theta=\frac{1}{3} \arccos \left(\frac{q^{\prime}}{2 \sqrt{-p^{\prime 3}}}\right) .
$$

\section{References}

[1] J.W. Dufty, in: Strongly Coupled Plasmas, Eds. F. Rogers, H.E. DeWitt, Plenum, New York 1987, p. 493.

[2] J.W. Dufty, L. Zoghaib, in: Strongly Coupled Plasmas, Ed. S. Ichimaru, Elsevier Sci., New York 1990, p. 533. 
[3] I.R. MacDonald, P. Vieillefosse, J.P. Hansen, Phys. Rev. Lett. 39, 271 (1977).

[4] A. Alatsuey, J. Lebowitz, D. Levesque, Phys. Rev. A 43, 2673 (1991).

[5] J.W. Dufty, L. Zoghaib, Phys. Rev. E 47, 2958 (1993).

[6] B. Held, P. Pignolet, J. Phys. (France) 47, 437 (1986).

[7] D. Boercker, C.A. Iglesias, J. Dufty, Phys. Rev. A 36 , 2254 (1987).
[8] M.T. Meftah, T. Chohra, H. Bouguettaia, F. Khelfaoui, B. Talin, A. Calisti, J.W. Dufty, Eur. Phys. J. B 37, 39 (2004).

[9] M. Berkovsky, J.W. Dufty, A. Calisti, R. Stamm, B. Talin, Phys. Rev. E 51, 4917 (1995).

[10] G.D. Harp, B.J. Berne, Phys. Rev. A 2, 975 (1970). 